\title{
Maximum principles for some quasilinear
}

\section{degenerate elliptic-parabolic operators}

\section{Margaret Anne Dow}

I combine methods of Bony [1], Hill [2], and Redheffer [5] with methods of Pucci [3, 4] and Výborný [6] to prove maximum principles and boundary principles for a classical solution to a quasilinear degenerate elliptic-parabolic inequality of the form

$$
\begin{aligned}
E u \equiv \sum_{k, I=1}^{m} a_{k l}(x, u, \bar{D} u) \bar{D}_{k} \bar{D}_{1} u+\sum_{i=1}^{n} b_{i}(x, u, \bar{D} u) D_{i} u+ & \\
& +d(x, u, \bar{D} u) u+a(x, u, \bar{D} u) \geq 0
\end{aligned}
$$

on a domain whose boundary satisfies a weaker condition than the interior sphere property. The operator is that of Redheffer except for the addition of a term $d u$ and for the appearance of nonlinearity in the coefficients of the partial derivatives $D_{i}$. The operator $\bar{D}_{k}$ is a linear combination of the derivatives $D_{i}$ with Lipschitz continuous coefficients.

The concepts of diffusion and drift trajectories, introduced by $\mathrm{Hill}$ and exploited further by Redheffer, lead to a definition of a propagation set for each point of the domain and its boundary. Here, however, drift trajectories are integral curves of the vector field $X(x)=\left(b_{1}(x, u, 0), \ldots, b_{n}(x, u, 0)\right)$. Under conditions similar to those of Pucci for linear operators and those of Výborny for quasilinear operators, I show that a non-negative interior maximum relative to the appropriate propagation set spreads along diffusion trajectories passing through that point. For operators of elliptic and parabolic type, such maxima also spread along drift trajectories in the direction of their

Received 27 October 1972. Thesis submitted to the University of Queensland, October 1972. Degree approved, March 1973. Supervisor: Professor R. Výborný. 
orientation. Thus such maxima spread to the propagation set in these two special cases.

The theory leads to boundary maximum principles describing the behaviour of the quotient

$$
\frac{u(x)-u(y)}{|x-y|}
$$

as $x$ approaches a boundary point $y$ along various paths.

These results imply that solutions to certain mixed boundary value problems are unique. All of the theorems can be extended to weakly coupled systems of inequalities.

Finally, I derive some three-circles theorems for the operator $E$.

\section{References}

[1] Jean-Michel Bony, "Principe du maximum, inégalité de Harnack et unicité du problème de Cauchy pour les opérateurs elliptiques dégénérés", Ann. Inst. Fourier (Grenoble) 19 (1969), 277-304.

[2] C. Denson HIII, "A sharp maximum principle for degenerate ellipticparabolic equations", Indiana Univ. Math. J. 20 (1970), 213-229.

[3] Carlo Pucci, "Proprietà di massimo e minimo delle soluzioni di equazioni a derivate parziali del secondo ordine di tipo ellittico e parabolico I", Atti Accad. Naz. Lincei Rend. Cl. Sci. Fis. Mat. Nat. (8) 23 (1957), 370-375.

[4] Carlo Pucci, "Proprietà di massimo e minimo delle soluzioni di equazioni a derivate parziali del secondo ordine di tipo ellittico e parabolico II", Atti Accad. Naz. Lincei Rend. Cl. Sci. Fis. Mat. Nat. (8) 24 (1958), 3-6.

[5] Ray Redheffer, "A sharp maximum principle for nonlinear inequalities", Indiana Univ. Math. J. 21 (1971), 227-248. 
[6] R. Výborný, "On a certain extension of the maximum principle", Differential equations and their opplications, 223-228 (Proc. Conf. Prague, September 1962. Publishing House of the Czechoslovak Academy of Sciences, Prague; Academic Press, New York, London, 1963). 